\title{
Audiometric notch as a sign of noise induced hearing loss
}

\author{
D I McBride, S Williams
}

\begin{abstract}
Objectives-To investigate the relation between different types of exposure to noise and a classic sign of noise induced hearing loss (NIHL), the audiometric notch.
\end{abstract}

Methods-The study sample had exposure to both continuous and impulse noise and was drawn from a population of electrical transmission workers. Audiograms, taken as part of a hearing conservation programme, were read by three clinicians experienced in the assessment of NIHL. Working independently and using their clinical judgment, they were asked to identify localised increases in the threshold of hearing (audiometric notches) which they would attribute to noise, had a suitable history of exposure been elicited. Prevalent cases of NIHL were identified by the presence of a notch in either ear. Risk factors for NIHL were assessed by a questionnaire which sought information about exposure to air blast circuit breaker noise; firearms; explosions, and continuous noise. The odds of exposure to these factors in those with and without hearing loss were calculated, and odds ratios (ORs) and $95 \%$ confidence intervals $(95 \%$ CIs) were estimated by logistic regression. Results-Of the 648 questionnaires sent out 357 were returned, a response rate of $55 \%$. Of these, at least two out of the three assessors identified $175(49 \%)$ people with a notch at any audiometric frequency. There was no association between these cases and the NIHL risk factors identified by the questionnaire, but a further frequency specific analysis showed a small proportion of people $(15 \quad(4 \%))$ with notches at $4 \mathrm{kHz}$ who had the expected associations with exposure to noise and a significant OR for firearms of $4.25(95 \%$ CI 1.28 to 14.1$)$. The much larger proportion of people with $6 \mathrm{kHz}$ notches $(110$ $(31 \%)$ ) did not show these associations. Conclusions-To diagnose NIHL it is important to elicit a detailed and accurate history of exposure to noise: although the notch at $4 \mathrm{kHz}$ is a well established clinical sign and may be valuable in confirming the diagnosis, the $6 \mathrm{kHz}$ notch is variable and of limited importance.

(Occup Environ Med 2001;58:46-51)

Keywords: hearing loss; noise induced; noise; occupational

Noise induced hearing loss has an insidious onset and may be well advanced by the time that it gives rise to appreciable disability. Early detection of such loss through audiometry may assist in prevention, and recognition of existing loss is important for educational and medicolegal purposes. Although the characteristic pattern of NIHL was recognised early in the development of pure tone audiometry, the progression of this loss was first studied systematically in cross sectional studies of the Jute industry in Dundee ${ }^{1}$ and in a cross section of general workplaces in England and Wales. ${ }^{2}$ These confirmed that with exposure to broad band, steady noise, or noise with an impulsive component, the first sign was a dip or notch in the audiogram maximal at $4 \mathrm{kHz}$ with recovery at 6 and $8 \mathrm{kHz}$. The notch broadens with increasing exposure, and may eventually become indistinguishable from the changes of aging (presbycusis), where the hearing shows a gradual deterioration at the high frequencies. Although $4 \mathrm{kHz}$ is the classic frequency affected the notch may be noted elsewhere because the frequency range of the noise influences where the cochlear damage occurs. The Health and Safety Executive note $A$ guide to audiometric testing programmes ${ }^{3}$ summarises the present practice guidelines on identifying NIHL, advising that:

\footnotetext{
"Assessing the low and high frequencies separately takes account of the fact that NIHL preferentially affects the high frequencies, with hearing loss beginning characteristically around $4 \mathrm{kHz}$ before spreading to the lower frequencies as the $4 \mathrm{kHz}$ loss progresses (the $4 \mathrm{kHz}$ dip). However, intense low frequency noise may cause maximal loss over the $0.5-2 \mathrm{kHz}$ range and intense high frequency noise $0.5-2 \mathrm{kHz}$ range and
loss at 6 or $8 \mathrm{kHz}$.
}

This suggests that the audiometric frequency where the notch occurs might be useful as a marker for specific types of exposure to noise. Clinical observation also suggested that the notch might be a useful early marker for exposure to noise. Salmivalli ${ }^{4}$ found that the notch begins at $6 \mathrm{kHz}$ twice as often as it begins at 4 $\mathrm{kHz}$; Axelsson ${ }^{5}$ was also of the opinion that the earliest change might be found at this frequency and a standard otolaryngological text ${ }^{6}$ advised that:

\section{"The very earliest changes in young subjects exposed to broad band noise for 1-2 years occur around $6 \mathrm{kHz}$. With a duration of exposure to noise of 2-5 years, noise induced permanent threshold shift (NIPTS) slides into the $4 \mathrm{kHz}$ region.”}

The frequency specific effect of different types of noise meant that the notch seemed ideal as an outcome measure in an epidemiological investigation which we were planning. The target population encountered continuous noise in the course of their work, but were also exposed to a particular type of impulse noise, that due to air blast circuit breakers. These are 
large switches in use in electricity transmission circuits which use a blast of high pressure air to extinguish the arc which forms when the circuit is broken. The peak sound level produced by this apparatus is of a magnitude in the order of that due to firearms and the peak impulse exposure to noise standard of $200 \mathrm{~Pa}$ (140 $\mathrm{dB}){ }^{7}$ Although the frequency range of air blast circuit breaker noise has a considerable low frequency component and should in theory be less harmful than exposure to firearms, the very long duration of the impulse is unlike any other form of noise. This gave rise to serious concerns about the risk of hearing loss in those exposed. In practice, however, there proved to be considerable problems with observer reliability in identification of the notch and as this paper reports there were also concerns that many of the notches identified did not seem to be strongly associated with any of the noise exposure factors identified in the study.

\section{Materials and methods}

STUDY SAMPLE

The study sample was drawn from the employees of an electricity transmission company which operates from locations throughout England and Wales. People who were exposed to impulse noise in excess of the peak exposure criterion of $200 \mathrm{~Pa}$ or who worked in designated hearing protection zones were referred to the occupational health service by location managers. At the time this study was carried out, 682 employees had been screened out of a total work force of 5628 .

Audiometry was carried out by nursing officers who had received training in audiometric techniques. The test followed the fixed frequency Békèsy protocol in accordance with BS $5966^{8}$ with a Bilsom CA850 audiometer with TDH-39 earphone and MX41/AR cushion combination. Tests were carried out in audiometric booths, and subjects were not exposed to noise in the 16 hours before the test. Calibration of the audiometers was carried out in accordance with the recommendations of ISO $389 .{ }^{9}$ The pure tone hearing threshold levels were recorded by the microprocessor control of the audiometer, and these thresholds were transferred to the audiometric record. At least one audiogram was available for each participant, and if more than one was available the most recent test was selected.

\section{AUDIOMETRIC ASSESSMENT}

Three raters with experience in assessment of audiograms, an otolaryngologist, an occupational physician with audiological training, and a senior audiometrician, were recruited to assess the audiograms. They were asked to inspect each audiogram and decide whether or not a notch was present, a notch which, if there were an appropriate history of exposure, they would attribute to the effects of noise. If so, they marked the location of the notch on the audiometric record. The results of this assessment have been reported elsewhere ${ }^{10}$ and showed that there was considerable variability in agreement between the assessors, the proportion of people identified as having a notch varying between 0.35 and 0.65 depending on the assessor. A chance corrected index of agreement was also calculated, in which 0 indicated no agreement and 1 indicated perfect agreement. The "all rater" index of agreement was 0.45 , and the range for pairwise comparisons was $0.14-0.52$ : therefore there was reasonable certainty that a person had a notch if at least two of the three assessors agreed.

\section{HISTORY OF EXPOSURE TO NOISE}

A personal dosimetry survey was carried out on a sample of 30 employees, selected to include a cross section of all the jobs with exposure to noise. This indicated that a small but notable percentage of the workforce was exposed to hazardous noise: seven people $(23 \%)$ had exposures above the first action level of 85 $\mathrm{dB}(\mathrm{A})$, four of these being above the second action level of $90 \mathrm{~dB}(\mathrm{~A})$. Although exposure to noise in the industry had remained relatively stable there were problems with applying these data retrospectively as most employees had held jobs outside the electricity supply industry. Noise exposure history was therefore collected by a specially designed questionnaire. Because the target group were dispersed throughout the country and many were peripatetic, this questionnaire was posted to their home address, with one follow up in case of non-response. Questions were asked about occupational exposure to steady state noise from machinery and noisy tools, exposure to air blast circuit breaker impulses, impulse noise from firearms, and traumatic exposure to explosive impulses. Noise exposure in employment was assessed from the job history by asking three questions about each job held. These were about the wearing of hearing protection, having to shout at work, and the use of noisy tools and equipment. Each of these questions had a binary response: yes or no for hearing protection and having to shout, occasionally or all the time for noisy tools. Three risk categories for continuous noise were identified: low risk where a respondent did not have to shout at work; intermediate risk where a person had to shout at work, but wore hearing protection; and high risk where a person had to shout at work and did not wear hearing protection.

Exposure to air blast circuit breakers was assessed by asking which, if any, jobs exposed them to air blast circuit breaker impulses and if so how many unprotected exposures they experienced.

Exposure to guns and firearms was assessed by asking about the conditions under which exposure took place, whether civilian or military; the weapon type; the duration of exposure; the total number of rounds fired for each type of weapon (with options of $0-100$, $100-1000$, or greater than 1000 rounds); and whether or not hearing protection had been used. Exposure to traumatic impulse was assessed by asking about experience of explosive events including the nature of the event. 
Table 1 Depth and shape of audiometric notches identified by at least two assessors

\begin{tabular}{lllcc}
\hline $\begin{array}{l}\text { Depth } \\
(d B)\end{array}$ & $\begin{array}{l}\text { Shape of } \\
\text { notch }\end{array}$ & $\begin{array}{l}\text { Two of three } \\
\text { assessors agree } \\
(n)\end{array}$ & $\begin{array}{l}\text { All agree } \\
(n)\end{array}$ & $\begin{array}{l}\text { Total of all } \\
\text { notches } \\
(n(\%))\end{array}$ \\
\hline $1-15$ & Narrow & 42 & 7 & $49(19)$ \\
& Wide & 12 & 2 & $14(6)$ \\
$16-30$ & Narrow & 42 & 28 & $70(28)$ \\
& Wide & 35 & 18 & $53(21)$ \\
$\geqslant 31$ & Narrow & 8 & 25 & $33(13)$ \\
& Wide & 11 & 24 & $35(14)$ \\
Total & & 150 & 104 & 254 \\
\hline
\end{tabular}

ETHICS

The project had ethical approval from the research ethics committee of the South Birmingham Health Authority.

STATISTICS

The relation between these factors and the notch was examined by calculating the odds of exposure for those in the notch and normal hearing categories. Odds ratios (ORs) and 95\% confidence intervals (95\% CIs) were calculated with the Egret software.

\section{Results}

Out of the total of 648 questionnaires sent out, 357 were returned, an overall response rate of $55 \%$. In the whole group (responders and nonresponders) two out of the three assessors identified 320 people with a notch, a prevalence of $49.4 \% ; 175$ of these were in the group who responded, a prevalence among responders of $49 \%$. The entire population was male.

In practice audiograms are a complex shape, but two audiometric features, the depth at the frequency of the notch and the shape of the curve, were chosen to represent audiometric configuration. The frequency at which the notch occurred had already been identified by the assessors, and the depth of notch at that frequency was calculated by subtracting the average value of hearing threshold level at the next higher and lower frequencies. Audiometric shape was classified as narrow or "V shaped" if there was a single frequency in the depth of the notch, and wide or "U shaped" if there was more than one frequency. Table 1 shows the distribution of these features. There were 152 narrow notches and 102 wide notches. Most notches (123 (49\%)) had a hearing threshold level 16-30 $\mathrm{dB}$ deep, with about equal numbers shallower and deeper than this $(63(25 \%)$ and $68(27 \%)$, respectively).

The duration of jobs in the electricity supply industry was short with a median of 9 years, although the median duration of jobs within the industry was much longer at 25 years. We examined the risk of hearing loss in the job held for the longest period in which there were prevalence ORs of 200, 103, and 54 in the low, medium, and high exposure categories respectively.

Unprotected ABCB exposure had a modal frequency of eight events, but there was a long upper tail to the distribution with $25 \%$ of the sample experiencing more than 20 exposures.

The modal frequency of firearms exposure varied according to the service and type of enlistment (navy, army or air force, as either a territorial/volunteer reservist or a regular serviceman). For territorial army service, the modal value was 100 rounds, reported by 12 people, for the regular army it was 1000 rounds, and 24 people had a similar finding for civilian gun clubs. The 12 national servicemen had a modal exposure value of 100 rounds, and for the navy and air force there were fewer respondents with modal exposure values of 1000 rounds for six naval men and 100 rounds for seven in the air force.

The ORs for exposure to the factors that might lead to hearing loss are reported in table 2. Most of the ORs were close to, and none were significantly different from, one. As this lack of association was not expected, we examined the relation between the variables in exposure to noise and notches at specific frequencies. As hearing loss may be asymmetric, there are several possible permutations, the frequencies of which are shown in table 3. Notches at $6 \mathrm{kHz}$ were most often identified, and unilateral $6 \mathrm{kHz}$ notches were found to be as prevalent as symmetric notches. Notches at $4 \mathrm{kHz}$ were fewer, but asymmetry was also common. This asymmetry meant that some men had notches at different frequencies in the two ears, so to ensure mutually exclusive groups the analysis was restricted to those with symmetric or unilateral notches at the relevant frequencies. Because the numbers of 3 and 4 $\mathrm{kHz}$ notches were few, analyses were carried out when a notch was present in either ear.

The only increased OR in the $3 \mathrm{kHz}$ category was for medium exposure to noise, OR 1.97 (95\% CI 0.39 to 9.94 ). The remaining results are shown in table 4 . The cases with a $4 \mathrm{kHz}$ notch are associated with the noise exposure variables, with raised ORs in all categories, and a significantly increased OR for firearms exposure. By contrast, the only increased OR in the

Table 2 Association between variables of exposure to noise and hearing loss according to the notch criterion

\begin{tabular}{|c|c|c|c|c|}
\hline Variable & $\begin{array}{l}\text { Normal } \\
\text { hearing } \\
\text { (n) }\end{array}$ & $\begin{array}{l}\text { Notch } \\
(n)\end{array}$ & $O R$ & $95 \% C I$ \\
\hline \multicolumn{5}{|c|}{ Exposure to noise in employment: } \\
\hline Low & 101 & 99 & 1.00 & Reference \\
\hline Medium & 51 & 52 & 1.04 & 0.64 to 1.67 \\
\hline High & 30 & 24 & 0.82 & 0.45 to 1.49 \\
\hline \multicolumn{5}{|c|}{ Exposure to air blast circuit breaker: } \\
\hline None & 27 & 20 & 1.00 & Reference \\
\hline Protected & 27 & 23 & 1.15 & 0.52 to 2.57 \\
\hline Unprotected & 128 & 132 & 1.39 & 0.74 to 2.61 \\
\hline \multicolumn{5}{|c|}{ Exposure to firearms: } \\
\hline Never exposed & 114 & 115 & 1.00 & Reference \\
\hline Ever exposed & 68 & 60 & 0.87 & 0.57 to 1.35 \\
\hline \multicolumn{5}{|c|}{ Exposure to an explosion: } \\
\hline Never exposed & 159 & 147 & 1.00 & Reference \\
\hline Ever exposed & 23 & 28 & 1.32 & 0.73 to 2.39 \\
\hline
\end{tabular}

Table 3 Distribution across ears, by frequency, of notches identified by at least two assessors

\begin{tabular}{llllc}
\hline \multirow{5}{*}{ Left $(\mathrm{kHz})$} & \multicolumn{2}{l}{ Right $(\mathrm{kHz})$} & 4 & 6 \\
\cline { 2 - 5 } & No notch & 3 & 2 & 49 \\
\hline No notch & - & 3 & 0 & $4^{\star}$ \\
3 & 3 & 0 & 6 & $12^{\star}$ \\
4 & 5 & $1^{\star}$ & $7^{\star}$ & 45 \\
\hline
\end{tabular}

^Indicate asymmetric hearing loss, so excluded from frequency specific analysis. 
Table 4 Association between variables in exposure to noise and a notch at 4 and $6 \mathrm{kHz}$

\begin{tabular}{|c|c|c|c|c|c|c|c|c|c|c|}
\hline $\begin{array}{l}\text { Type of exposure to } \\
\text { noise }\end{array}$ & $\begin{array}{l}\text { No } \\
\text { notch }\end{array}$ & $4 \mathrm{kHz} z^{\star}$ & OR & $95 \% C I$ & $6 \mathrm{kHz}+$ & OR & $95 \% C I$ & $\begin{array}{l}6 \mathrm{kHz} \\
\text { both ears }\end{array}$ & $O R$ & $95 \% C I$ \\
\hline \multicolumn{11}{|l|}{ Continuous: } \\
\hline Low & 103 & 6 & 1.00 & Reference & 63 & 1.00 & Reference & 28 & 1.00 & Reference \\
\hline Medium & 54 & 5 & 1.65 & 0.49 to 5.54 & 32 & 0.97 & 0.57 to 1.66 & 12 & 0.82 & 0.39 to 1.73 \\
\hline High & 31 & 3 & 1.90 & 0.46 to 7.87 & 15 & 0.79 & 0.40 to 1.58 & 5 & 0.59 & 0.21 to 1.67 \\
\hline \multicolumn{11}{|l|}{ Impulse: } \\
\hline None & 27 & 1 & 1.00 & Reference & 13 & 1.00 & Reference & 6 & 1.00 & Reference \\
\hline Protected & 27 & 4 & 4.00 & $\begin{array}{l}0.43 \text { to } \\
37.17\end{array}$ & 12 & 0.92 & 0.36 to 2.38 & 7 & 1.17 & 0.35 to 3.92 \\
\hline Unprotected & 134 & 9 & 1.65 & $\begin{array}{l}0.20 \text { to } \\
13.33\end{array}$ & 85 & 1.32 & 0.64 to 2.69 & 32 & 1.07 & 0.41 to 2.82 \\
\hline \multicolumn{11}{|l|}{ Firearms: } \\
\hline Never exposed & 118 & 4 & 1.00 & Reference & 79 & 1.00 & Reference & 28 & 1.00 & Reference \\
\hline Ever exposed & 70 & 10 & 4.77 & $\begin{array}{l}1.46 \text { to } \\
15.53\end{array}$ & 31 & 0.66 & 0.40 to 1.10 & 17 & 1.02 & 0.52 to 2.00 \\
\hline \multicolumn{11}{|l|}{ Explosion: } \\
\hline Never exposed & 164 & 11 & 1.00 & Reference & 95 & 1.00 & Reference & 36 & 1.00 & Reference \\
\hline Ever exposed & 24 & 3 & 1.68 & 0.45 to 6.23 & 15 & 1.08 & 0.54 to 2.16 & 9 & 1.71 & 0.73 to 3.98 \\
\hline
\end{tabular}

*A $4 \mathrm{kHz}$ notch present in either or both ears.

†A $6 \mathrm{kHz}$ notch present in either ear.

$6 \mathrm{kHz}$ notch category was for exposure to an explosive event and this was not significant.

\section{Discussion}

The notch has long been recognised as a clinical sign of exposure to noise, and although the classic association is between continuous exposure to noise and a notch at $4 \mathrm{kHz}$, notches have been also been observed at $6 \mathrm{kHz}$ in people exposed to impulse noise and at $3 \mathrm{kHz}$ with low frequency noise. Such exposure was prevalent in this sample, with most $(310(86 \%))$ exposed to air blast circuit breaker impulse noise and a considerable proportion (128 $(36 \%))$ exposed to firearms. We had a reasonable expectation that these exposures would account for the relatively high prevalence of audiometric notches in this sample-but found no association between the two. Further investigation showed that this was due to a lack of association between noise exposure factors and the $6 \mathrm{kHz}$ notch: the separate analysis of $4 \mathrm{kHz}$ notches showed the expected outcomes, with a significant OR for firearms, and ORs for the other factors which were deviated, if not significantly so. We sought possible explanations. As audiometric variability is greater at 6 than at $4 \mathrm{kHz}^{11}$ it was possible that the $6 \mathrm{kHz}$ notches were transient, and caused by chance. For 102 men in our sample two audiograms were available, allowing us to check that the notches persisted. For $4 \mathrm{kHz}$ notches, 15 out of $29(52 \%)$ were detected at the second test, whereas 50 of $73(68 \%) 6 \mathrm{kHz}$ notches persisted. The main reason for the change was that $6 \mathrm{kHz}$ notches had become $4 \mathrm{kHz}$ notches and vice versa. Audiometric variability was therefore present but does not seem to have a disproportionate effect at the $6 \mathrm{kHz}$ frequency.

Another explanation was that the exposure was insufficient to cause a notch; however, both types of impulse exposure seemed to be clinically important. Air blast circuit breakers are operated manually during routine switching, and at typical operating distances of about 10 metres, exposures in excess of the peak exposure standard of $200 \mathrm{~Pa}$ are quite likely, with median peak levels in the order of $632 \mathrm{~Pa}$ $(150 \mathrm{~dB})$. Occasional unexpected operation could also take place during faults, possibly close to the workers, which would result in a more intense exposure. In common with many other organisations the use of hearing protection did not become compulsory until the enactment of the Noise at Work Regulations, ${ }^{12}$ so that most of the sample had unprotected exposure. Although there was a low modal value of exposure at eight, the long upper tail meant that $25 \%$ of the sample had 20 or more exposures. Had these exposures been sufficient to cause acoustic trauma, an effect would have been expected, especially perhaps at the low audiometric frequencies.

In this sample the most often used firearms were British service issue weapons including the .303 Lee Enfield rifle and the $7.62 \mathrm{~mm}$ self loading rifle, both with peak exposures in the order of $2 \mathrm{kPa}(160 \mathrm{~dB})$. Self reporting of the number of rounds fired seemed to be in line with exposures that would be expected. Modal duration of service for territorials was 1 year, and during this period recruits might have one or two range practices, shooting five or 10 10-round magazines on each occasion. For regular servicemen, exposure was somewhat greater possibly due to a greater duration of exposure. Although the number of rounds does not seem high, the values are probably realistic and are in fact in line with operational data; for example during the Falklands war the modal value for personal exposure to weapons reported was 100 rounds. ${ }^{13}$

The results suggest that these clinically important exposures did not in fact have an adverse effect. Although exposure in excess of the $200 \mathrm{~Pa}$ peak exposure standard is often perceived to be harmful, this is not necessarily so. Impulse noise standards - such as that proposed by Coles and Rice ${ }^{14}$ — suggest that, providing the duration of the exposure is taken into account, exposures of up to $3.5 \mathrm{kPa}(165$ $\mathrm{dB})$ may be safe. Although the exposure to noise from air blast circuit breakers was very variable, a review of existing exposure data suggested that many exposure events would be safe or of borderline risk. For firearms, the British Defence Standard ${ }^{15}$ suggests that exposure of up to 60 rounds of ammunition in 24 hours is an acceptable hazard. This could probably be experienced several times a year 
without untoward effect, so that total exposures in the order of 100-1000 rounds may not give an excess risk of hearing loss.

As there were only 14 prevalent cases by hearing loss at $4 \mathrm{kHz}$, this suggests that the underlying attributable risk from the factors under consideration was probably not high. To investigate this, a confirmatory analysis was carried out with the Health and Safety Executive categorisation in which men are classified into warning or referral categories of hearing loss if the hearing threshold levels at the mean of the low $(0.5,1$ and $2 \mathrm{kHz})$ or high $(3,4$, and $6 \mathrm{kHz}$ ) audiometric frequencies exceed certain age adjusted levels. ${ }^{3}$ This placed 121 men in either the warning or referral categories of hearing loss. The results have been reported ${ }^{16}$ : ORs for exposure to an air blast circuit breaker were 2.27 (95\% CI 1.01 to 5.08) and 2.10 (95\% CI 0.97 to 4.54 ) for the protected and unprotected groups respectively, which does not suggest a high risk. For firearms, the OR was 1.76 (95\% CI 1.12 to 2.77 ), however, with an adjustment for age in four bands (18-30, $31-40,41-50$, and $>50$ ) this OR became nonsignificant. Because age, exposure to noise, and hearing threshold level are so strongly correlated it is difficult to place a definite interpretation on this result, but it argues against the importance of exposure to gunfire.

The effect of noise in this study does not seem to have been particularly strong, which lends support to the impulse noise standards, but even so, some men undoubtedly had considerable exposure to noise, as indicated by the association with the $4 \mathrm{kHz}$ notch: it was therefore reasonable to expect some association with the $6 \mathrm{kHz}$ notch, either as an early marker or a sign of exposure to impulse noise. The clinical findings already referred to were backed up by our data. In 1959 Gravendeel and Plomp ${ }^{17}$ reported using a method of continuous frequency testing on the hearing of many hundreds of soldiers exposed to light firearms. They found the "average place of the dips" to be $5.9 \mathrm{kHz}$, and emphasised that although a $\mathrm{C}^{5}(4 \mathrm{kHz})$ notch might be found, the maximum loss might lie anywhere between 6 and $8 \mathrm{kHz}$. This was confirmed by analysis of other data from the military setting - for example the report by Salmivalli that, with exposure to acoustic trauma, the greatest reduction of hearing appeared at $5.5 \mathrm{kHz} .{ }^{18}$

Because these studies did not include a control group care is required in their interpretation, but the findings with firearms noise tended to be confirmed by an impact noise study carried out in a sample of 511 drop forge workers, ${ }^{19}$ in which the mean binaural hearing threshold level of the exposed group showed a significant $6 \mathrm{kHz}$ notch compared with that of controls. This result is at odds with that of another survey in a shipyard which examined the hearing of controls and compared them with one group exposed to continuous noise, and groups with short, intermediate, and long periods of exposure to impulses of noise. ${ }^{20}$ The low and intermediate impulse groups had symmetric $6 \mathrm{kHz}$ notches, as had the control group. This study also showed that asymmetric hearing loss is not unusual, as the high impulse group had a $4 \mathrm{kHz}$ notch in the left ear and a 6 $\mathrm{kHz}$ notch in the right ear, while the continuous group had a $6 \mathrm{kHz}$ notch in the left and no notch on the right.

A possible unifying interpretation of these findings is that the $6 \mathrm{kHz}$ notch may be a common incidental finding unrelated to exposure to noise. This conjecture is supported by the data, if not the conclusions, from community studies in which the results have also been variable. A Canadian group ${ }^{21}$ tested a randomly selected sample from a student population, and found a high prevalence $(40 \%)$ of notches in one or both ears, with most of these at $6 \mathrm{kHz}$. The possible aetiological factors studied were music (listening to stereo and band music), use of machinery (snowmobiles, motorcycles, and chainsaws) and firearms (hunting and shooting), but the only significant correlations were between the notch and the factors associated with music. Axelsson et al ${ }^{2}$ reported a $15 \%$ prevalence of hearing loss of greater than $20 \mathrm{~dB}$ hearing threshold level at any frequency in teenage boys, with the highest proportion of these notches at $6 \mathrm{kHz}$. Although leisure exposure to noise was suspected to be responsible, the only marked relation found was:

"for a family history of hearing loss on the one hand and hearing loss in the left ear at 4,6 , and $8 \mathrm{kHz}$ on the other".

An important but often overlooked reason why the $6 \mathrm{kHz}$ notch is common relates to the standardisation of hearing. Human hearing sensitivity is not the same across the range of audiometric frequencies represented in the audiogram. As defined by the hearing of otologically normal young adults, this is (with respect to a reference level of $20 \mu \mathrm{Pa}) 27 \mathrm{~dB}$ at $250 \mathrm{~Hz}, 11.5 \mathrm{~dB}$ in the midrange $(3 \mathrm{kHz}), 16$ $\mathrm{dB}$ at $6 \mathrm{kHz}$, and $15.5 \mathrm{~dB}$ at $8 \mathrm{kHz} .{ }^{23}$ Although this should have the effect of normalising the shape of the audiogram so that it appears as a straight line, if, as Robinson suggests, the reference standard at $6 \mathrm{kHz}$ is set several $\mathrm{dB}$ too low $^{24}$ a normal audiogram would have a notch. This is confirmed by data from the national study of hearing ${ }^{25}$ in which the predicted hearing threshold levels show just such an effect.

It seems that the $6 \mathrm{kHz}$ notch may not be a good marker for high intensity exposure to noise, and because of the wide variety of audiometric shapes there are also considerable concerns about the reliability of notch identification. The main problem here seems to be that there is no standard definition of an audiometric notch, so people tend to develop their own criteria. ${ }^{10}$ This process involves pattern recognition and the selection of visual cues, but at a fairly simple level the process involves selecting a criterion for depth. This is part of the explanation for the lack of agreement in the study: one of the assessors selected only the deeper notches.

Whatever the reason the fact that this unreliability exists is unfortunate because the diagnosis of hearing loss due to exposure to noise is primarily an audiometric one. In the absence of a clinical marker the diagnosis must depend on 
an assessment of whether or not the loss in question is in excess of that which would be expected for age. As hearing is normally distributed (with a positive skew), this will be a purely arbitrary decision unless supported by an adequate assessment of the noise dose, with reference to the level and duration of the exposure experience of the person. If this information is available one of the reference standards such as the International Standards Organisation (ISO) $1990^{26}$ presents formulae from which can be calculated the expected hearing loss for any selected percentile of the population. Even then, as Hinchcliff says:

"The most one can say is that the audiometric findings are compatible, or not compatible, with the occupational exposure to noise history given by that individual".

The exposure to noise in this sample was clinically important, yet comparison with exposure standards showed the borderline nature of the risk. We conclude that to make a diagnosis of NIHL it is important to elicit a detailed and accurate history of exposure to noise: although the notch at $4 \mathrm{kHz}$ is a well established clinical sign and may be valuable in confirming the diagnosis, the $6 \mathrm{kHz}$ notch is variable and of limited importance.

1 Taylor W, Pearson J, Mair A, et al. Study of noise and hearing in jute weaving. 7 Acoust Soc Am 1965;38:113-20.

2 Burns W, Robinson DW. Hearing and noise in industry. London: Her Majesty's Stationery Office; 1970

3 Health and Safety Executive. A guide to audiometric testing programmes. London: The Stationery Office; 1995. (Guidance note no MS26.)

4 Salmivalli A. Military audiological aspects in noise-induced hearing losses. Acta Otolaryngol Suppl 1979;360:96-7.

5 Axelsson A. Diagnosis and treatment of occupational noiseinduced hearing loss. Acta Otolaryngol Suppl 1979;360:86-

6 Maran A, Stell P. Clinical otolaryngology. Oxford: Blackwell, 1979:102.

7 Health and Safety Executive. Noise at work: the Noise at Work Regulations 1989. London: The Stationery Office, 1989.

8 British Standards Institution. Specification for audiometers. London: British Standards Institution; 1980. (Standard No BS 5966.)
9 International Standards Organisation. Standard reference zero for the calibration of pure tone audiometers. Geneva: International Standards Organisation; 1964. (Standard No R389.)

10 McBride DI, Williams SM. Characteristics of the audiometric notch as a clinical sign. Scand Audiol (in press).

11 Atherley GRC, Dingwall-Fordyce I. The reliability of repeated auditory threshold determination. $\mathrm{Br} \mathcal{7}$ Ind $\mathrm{Med}$ 1963;20:231-5.

12 The Noise at Work Regulations 1989. London: The Stationery Office, 1989. (Statutory Instrument 1989 No 179.)

13 Brown J. Noise induced hearing loss sustained during land operations in the Falklands Islands Campaign, 1982. F Soc Occup Med 1985;35:44-54.

14 Coles RRA, Garinther GR, Hodge DC, et al. Hazardous exposure to impulse noise. F Acoust Soc Am 1968;43:33643.

15 Ministry of Defence. Acceptable limits for exposure to impulse noise from military weapons, explosives and pyrotechnics. Glasgow: Ministry of Defence; 1985. (Defence Standard No 00-27/1.)

16 McBride D, Williams S. Air blast circuit breaker noise and hearing loss: a multifactorial model for risk assessment. Occup Med (Lond) 2000;50:173-81.

17 Gravendeel DW, Plomp R. The relation between permanent and temporary noise dips. Arch Otolaryngol 1959;69:71419.

18 Salmivalli A. Acoustic trauma in regular army personnel. Clinical audiologic study. Acta Otolaryngol Suppl 1967;222: $1-85$.

19 Sulkowski WJ. Hearing impairment caused by impulse noise: survey in the drop forging industry. Scand Audiol Suppl 1980;12:307-17.

20 Mäntysalo S, Vuori J. Effects of impulse noise and steady state noise on hearing. Br f Ind Med 1984;41:122-32.

21 Lees RE, Roberts JH, Wald Z. Noise induced hearing loss and leisure activities of young people: a pilot study. Can $\mathcal{F}$ Public Health 1985;76:171-3.

22 Axelsson A, Jerson T, Lindberg U, et al. Early noise-induced hearing loss in teenage boys. Scand Audiol 1981;10:91-6.

23 International Standards Organisation. Standard reference zero for the calibration of pure tone air conduction audiometers. Geneva: International Standards Organisation; 1991. (Standard No ISO 389.)

24 Robinson DW. Threshold of hearing as a function of age and sex for the typical unscreened population. $\mathrm{Br} \mathcal{F}$ Audiol 1988;22:5-20.

25 Lutman ME, Spencer HS. Occupational noise and demographic factors in hearing. Acta Otolaryngol Suppl 1991; 476:74-84.

26 International Standards Organisation. Acoustics: determination of occupational noise exposure and estimation of noise induced hearing impairment. Geneva: International Standards Organisation, 1990. (Standard No ISO 1999.)

27 Hinchcliff R. Occupational noise induced hearing loss. In: Raffle PAB, Adams PH, Baxter PJ, Lee WR, eds. Hunter's diseases of occupations, 8th ed. London: Edward Arnold, 1994:282

\section{Correspondence and editorials}

Occupational and Environmental Medicine welcomes correspondence relating to any of the material appearing in the journal. Results from preliminary or small scale studies may also be published in the correspondence column if this seems appropriate. Letters should be not more than 500 words in length and contain a minimum of references. Tables and figures should be kept to an absolute minimum. Letters are accepted on the understanding that they be subject to editorial revision and shortening.

The journal also publishes editorials which are normally specially commissioned. The Editor welcomes suggestions regarding suitable topics; those wishing to submit an editorial, however, should do so only after discussion with the Editor. 